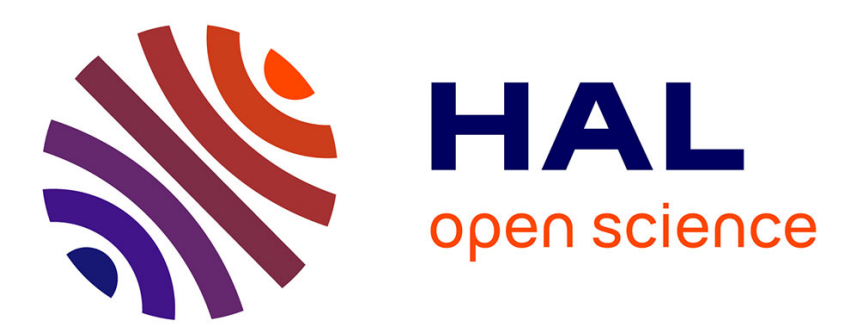

\title{
A Region-Based Progressive Coding of Semi-Regular 3-D Meshes for View-Dependent Transmission.
}

Céline Roudet

\section{To cite this version:}

Céline Roudet. A Region-Based Progressive Coding of Semi-Regular 3-D Meshes for View-Dependent Transmission.. 2010 Sixth International Conference on Signal-Image Technology and Internet-Based Systems (SITIS), Dec 2010, Kuala Lumpur, Malaysia. pp.51 - 59, 10.1109/SITIS.2010.19 . hal00589403

\section{HAL Id: hal-00589403 https://hal.science/hal-00589403}

Submitted on 28 Apr 2011

HAL is a multi-disciplinary open access archive for the deposit and dissemination of scientific research documents, whether they are published or not. The documents may come from teaching and research institutions in France or abroad, or from public or private research centers.
L'archive ouverte pluridisciplinaire HAL, est destinée au dépôt et à la diffusion de documents scientifiques de niveau recherche, publiés ou non, émanant des établissements d'enseignement et de recherche français ou étrangers, des laboratoires publics ou privés. 


\title{
A Region-Based Progressive Coding of Semi-Regular 3-D Meshes for View-Dependent Transmission
}

\author{
Céline Roudet \\ Université de Bourgogne, LE2I \\ Dijon, F-21078, France \\ Email: Celine.Roudet@u-bourgogne.fr
}

\begin{abstract}
We first introduce in this paper a new waveletbased segmentation algorithm for three-dimensional (3-D) Semi-Regular (SR) meshes. This method is then considered as a pre-processing step in a view-dependent progressive coding of 3-D meshes. Our segmentation process aims at producing homogeneous regions which have similar frequency amplitudes on the mesh surface, in other words: patches with different degrees of roughness. As a preliminary step of the development of a locally-based Rate-Distortion (R-D) optimized coding scheme, we propose to study the behaviour of the wavelet decomposition in the created patches, during the coding and the view-dependent reconstruction processes. To our knowledge, no previous work has ever considered the influence of the nonrefined parts of a mesh on the more detailed ones, in a viewdependent context. The main contribution of this paper consists in considering three different possible wavelet decompositions, close to the patch borders, and to study their influence during the coding and the view-dependent reconstruction stages. Among these three decompositions, we define a new scheme and finally propose various experimentations to demonstrate that it behaves better than the other classical considerations, for view-dependent reconstruction purposes.
\end{abstract}

Keywords-Geometric wavelets; multiresolution analysis; lifting scheme; progressive mesh compression; progressive coding; 3-D mesh segmentation; view-dependent reconstruction.

\section{INTRODUCTION}

Three-dimensional (3-D) objects and scenes find their way into more and more applications, thanks to the ever increasing bandwidth available in modern telecommunication networks, such as the High Speed Internet, or 3G mobile networks. These kinds of models are not only used in medical applications and entertainment (video games or animation films), but also for industrial purposes like car industry (Computer Aided Design framework), simulation or virtual environment contexts.

The triangular mesh is actually the most common representation for these objects and scenes, because it is a suitable model for many applications, like rendering for example. It includes geometry (vertex positions) and connectivity data which can be expensive in terms of computation, storage, transmission and rendering, even with today devices and networks.

Consequently in literature, many progressive mesh compression and transmission schemes have been proposed (including [1] - [8]), to represent data with multiple Levels of Detail (LOD). With this representation, a coarse approximation can subsequently be further improved depending on the user's devices (network channel and visualization terminal) and expectactions. This scalable representation is commonly obtained with a wavelet transform ([4] - [8]), because it produces a good decorrelation of the data. The best compression rates on 3-D meshes are currently obtained with a wavelet decomposition, also called Multi-Resolution Analysis (MRA) on Semi-Regular (SR) structures. In SR meshes, the valence of most vertices is equal to six, which produces a very compact representation of the connectivity information.

Most of the state-of-the-art wavelet-based SR mesh compression algorithms ([4] - [7]) consider a global wavelet decomposition on the mesh surface. Since the 3-D application expectations in terms of realism are always growing, it requires increasingly complex and large polygonal meshes. Consequently view-dependent compression, transmission and rendering methods ([8] - [11]) have recently emerged, to accelerate the huge amount of computations involved in this context. The principle consists in considering the user's viewing frustrum, to more refine the object regions or scene parts that face toward the viewer. Hoppe [11] was one of the formest to introduce the concept of view-dependent simplification and refinement, for fast and efficient rendering of 3-D irregular meshes (represented with the Progressive Mesh (PM) [1] structure).

More recently, several view-dependent compression and transmission algorithms have been developped for SR meshes [8] - [10]. Gioia et al. [9] proposed a waveletbased view-dependent transmission and visualization algorithm where the wavelet coefficients can be added or suppressed in real-time on a partially reconstructed mesh. Hence the reconstruction of large meshes can be optimized by allocating a major portion of the available bandwidth to visible parts.

Sim et al. developped in 2005 a rate-distortion (R-D) optimized view-dependent SR mesh streaming method [8]. One of the key points is to add a partitioning of the SR mesh into many segments, in order to optimally allocate bits during the compression process. Another advantage is 
to give the possibility to progressively transmit the segments independently, according to the client's viewing position. This region-based R-D optimization scheme improves the conventional wavelet-based compression algorithms for SR meshes ([4], [5]). The authors considered the same constrained allocation problem as in [6] and [7], to formulate their R-D optimization. Moreover, the server can allocate an adaptive bitrate to each segment (based on its visibility priority), to facilitate the interactive streaming of 3-D data. More recently, another view-dependent geometry coding of static 3-D scenes (defined by sets of semi-regular meshes) was proposed by Payan et al. [10]. The proposed coding scheme combines a segmentation for determining the visible regions, and an allocation process (detailed in [7]) for improving the visual quality of the encoded scene.

These two previously cited region-based view-dependent coding methods [8] and [10] define two different segmentations: the first one is fixed during the encoding process, whereas the second one is computed for a determined viewing frustrum. In this paper, we introduce a new SR mesh segmentation algorithm (adapted from the one detailed in [12]). We also propose to study the behaviour of the wavelet decomposition in the created regions, during the coding and the view-dependent reconstruction processes. Our segmentation process can be seen as a pre-processing step and is not dependent on the user's viewing position. It aims at producing homogeneous regions which share similar frequency amplitudes on the mesh surface, in other words: regions with different degrees of roughness.

None of the two previous algorithms ([8] and [10]) and no other paper (to our knowledge) has ever considered the influence of the non-refined parts of a mesh on the more detailed ones, in a view-dependent context. In the wavelet-based SR mesh compression setting, a subdivision scheme is used as a prediction operator during the MRA, to reduce the details lost during this coarsification process (regarded as wavelet coefficients). The most used prediction scheme for SR meshes is the Butterfly subdivision stencil [13] (illustrated in Fig. 1 (picture $(b)$ ). The main contribution of this paper consists in considering three different possible applications of this stencil, close to the patch borders, and to study their influence during the coding and the view-dependent reconstruction processes. We propose various experimentations to demonstrate that, among these three schemes, the one we have developped can reduce the bandwidth requirement for 3-D mesh view-dependent transmission.

The remainder of this paper is organized as follows. We begin by an explanation of the MRA on meshes in Section II, which produces the wavelet coefficients. The wavelet-based segmentation algorithm we developped is then described in Section III. The different patch-aware applications of the Butterfly prediction are detailed in Section IV. Experimental results are presented in Section $\mathrm{V}$, together with possible applications of our framework. As a conclusion, discussions and ideas for future work are given in the last section.

\section{MR ANALYSis ON SR MESHES}

In this section, we first detail the theory involved to produce wavelet coefficients on SR structures, with a lifting scheme formulation (second generation wavelets). A SR mesh is obtained thanks to a remeshing stage, which consists in resampling an input model (with irregular connectivity). We present then the concept of the state-of-the-art SR remeshing and MRA algorithms. The MRA produces a reversible mesh decomposition into a series of approximation meshes (called "hierarchy" hereinafter) and a sequence of details: the wavelet coefficients. We finally describe the averaging rule we applied to represent all the details lost during this simplification process, in only one coarse mesh of the "hierarchy".

\section{A. Wavelet Theory and Lifting Scheme}

Mallat first introduced in 1989 [14] the dyadic MRA and linked the wavelet transform to the filter bank theory. This MRA formulation, defined for finite energy functions, is formally described for a series of imbricated subspaces $\left(V^{m}\right)_{m \in \mathbb{Z}} \subset L^{2}(\mathbb{R})$ in which an orthonormal scaling function basis is determined. The orthogonal projection of a discrete signal $s(t)$ into the scaling function family forms a set of approximations, obtained by the application of a lowpass filter, followed by a uniform subsampling. To recover the high frequencies lost after the application of the lowpass filter (for any approximation in the level $i$ ), we have to take into account the $W^{i}$ space, which is the orthonormal complement of the $V^{i}$ space in $V^{i-1}$. These wavelet coefficients are hence obtained by a highpass filtering.

Considering the filter bank theory, it's not always possible to construct analysis tools with good properties for compression purposes. Consequently most of the methods have benefited from the lifting scheme formulation, introduced by Sweldens [15]. It moreover reduces computational costs and memory allocation, by first splitting the signal ( $S$ operator in Fig. 1, picture $(a)$ ) into even and odd components by using lazy wavelets. The following lifting steps ( $P$ and $U$ operators in Fig. 1, picture $(a)$ ) produce a modification of this lazy wavelet basis in order to add the desired properties. These operations can be simplified thanks to the lifting formulation with finite filters. Hence, the multi-resolution synthesis is simply obtained by an inversion of the order and sign of the analysis lifting operators.

The most used prediction operator $(P)$ on SR meshes (the Butterfly scheme [13]) is illustrated in Fig. 1 (picture $(b)$ ). The update operator $(U)$ is used to preserve the mean value of the signal. For compression purposes, the decomposition ends up with a normalisation step $(N)$, which is used to 


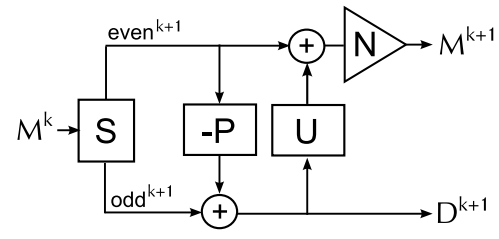

(a)

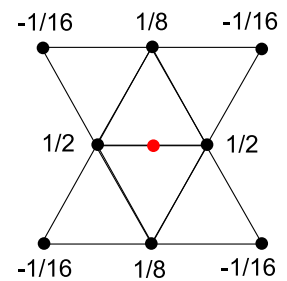

(b)
Figure 1. (a) Principle of the lifting scheme for the decomposition of a fine mesh $\left(M^{k}\right)$ into a coarser one $\left(M^{k-1}\right) . D^{k+1}$ represents the details lost during the simplification. (b) The most used prediction operator on SR meshes: the Butterfly subdivision stencil [13], which allows to predict the position of each newly added (odd) vertex (in red) thanks to its coarser (even) neighboors.

adjust the scale between the odd and the even coefficients, so as to decompose the surface in a normalised basis.

One of the lifting scheme advantages is that it can be applied to irregular point sets like meshes. In practice, we use a resampling of the irregular meshes (known as remeshing), to regularize the connectivity and to benefit from a more compact representation of the connectivity data.

\section{B. Remeshing and Wavelets on Meshes}

MR analysis for triangle meshes with arbitrary topology was introduced by Lounsbery [16] who demonstrated that a subdivision scheme can serve as a scaling function basis to extend the wavelet theory for irregular sampled signals like meshes. The compression performances are then highly dependent on the quality of the parameterization defined during the SR remeshing.

The main SR remeshing algorithms implemented in this context [17], [18], [19], rely on the same philosophy. They aim at reducing the parametric information (tangential set of wavelet coefficients), to represent details only with their geometric attributes. For that purpose, a mesh simplification is used to produce a coarse mesh (called the base complex). The challenge consists in minimizing the distortion produced during the parameterization of the input model, when mapping each curved surface to its associated coarse triangle in the base complex. Finally, the resampling stage used to produce the SR mesh from the base complex may vary, but is always obtained with subdivision surfaces.

The Normal Mesh algorithm [18] we considered to obtain the SR models presented in this work, applies a recursive piercing procedure and unlifted Butterfly wavelets to concentrate the high-frequency information along the surface normal. The resulting meshes are ideally suited for progressive compression purposes, because almost all of the geometric details are expressed with a single scalar. This algorithm currently produces one of the best remeshes for compression purposes, but only for closed surfaces.

Given SR meshes with the fewest connectivity and parametric data to encode, efficient data structures and process-

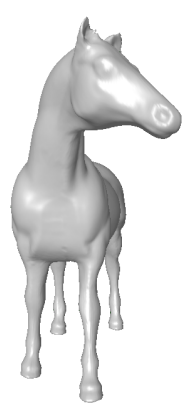

(a)

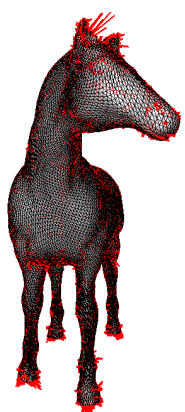

(b)

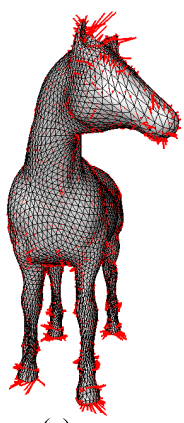

(c)

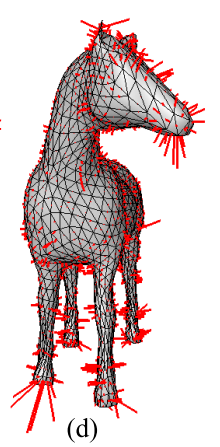

(d)
Figure 2. (a) Original Normal Horse model; (b-d) Wavelet coefficients represented as 3-D vectors (in red) linked to the edges of the first three decompositions (multiplication factor used to emphasize them: 10).

ing algorithms are used to get closer to the methods used for data sampled on regular grids. The application of the MRA on SR meshes, resulting from these latter algorithms, and mainly used for progressive compression purposes, can be based on various subdivision schemes. Most of the existing methods have benefited from interpolating subdivision filters (and in the most cases from the Butterfly stencil) for the lowresolution versions to be good approximations of the original object (in a least-squares sense).

Each mesh refinement, processed during the MR synthesis, is based on canonical quadrisections applied on every facet. This one-to-four triangle construction consists in adding three new vertices in the middle of each coarse edge. The prediction operation followed by the wavelet coefficient addition enable to recover the real position of these newly added vertices. Consequently the common representation of the wavelets consists in associating them with their corresponding coarse edges, as we can see in Fig. 2, on the first three decompositions of the Horse model.

Most of the actual state-of-the art mesh compression methods ([4] - [8]) apply a global wavelet decomposition (same lifting filters on the entire surface). Since the considered prediction schemes generate smooth finite surfaces, another kind of prediction, quantization and/or coding could be examined for the non-smooth parts of the mesh. We developped our segmentation process in this sense and considered only Normal meshes in our study, since they have been proven to provide the best compression rates.

\section{Wavelet Coefficients seen as a Segmentation Criterion}

We propose to study the wavelet coefficient amplitude distribution in the "hierarchy" produced by a global MRA. Our goal is to create regions on the mesh surface with different degrees of roughness: reflected by the wavelet coefficient amplitude. We illustrate in Fig. 3, 4 and 5 the normalized distribution of the coefficient amplitude, on various resolution levels of the Horse and Venus models (remeshed by the Normal Mesh [18] algorithm). 


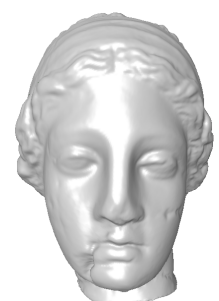

(a)

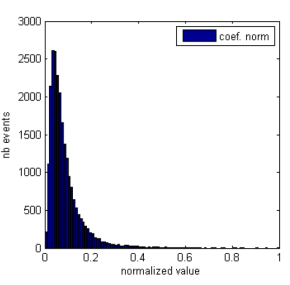

(b)

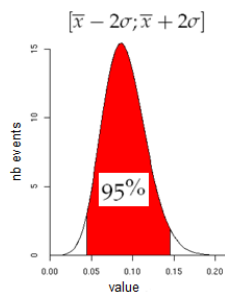

(c)

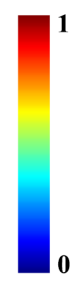

(d)
Figure 3. Distribution of the wavelet coefficient norm, on the first resolution level of the Normal Venus model. (a) Original model; (b) Histogram of the normalized coefficient norms associated to every resolution level; (c) The Gaussian normalization method we used to define a confidence interval (in red); (d) Color scale used in this figure and the next ones.

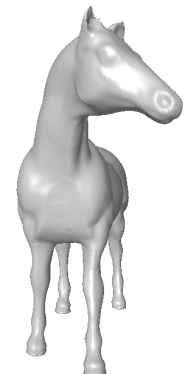

(a)

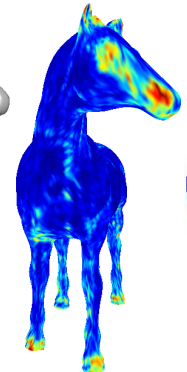

(b)

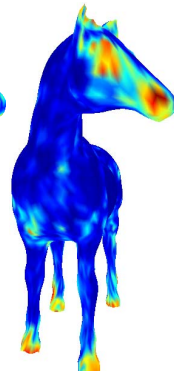

(c)

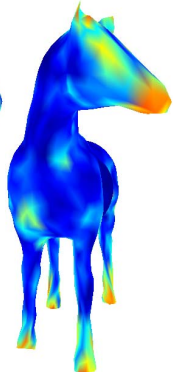

(d)

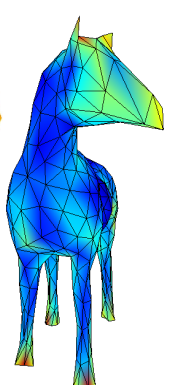

(e)
Figure 4. Gaussian normalized distributions of the wavelet coefficient norm, on the first four resolution levels of the Normal Horse model.

We applied a Gaussian normalization on the amplitude distributions (represented in Fig. 3, picture (c)), whose goal is to narrow extreme values. It hence allows to easier classify and segment a mesh with the considered criterion, since we can retain only the most represented samples. The application of this statistical technique (known as a confidence interval) is possible because the related histogram (depicted for the Venus model in Fig. 3, picture $(b)$ ) has a Gaussian-like normal distribution. Given the distribution averaging $(\bar{x})$ and the standard deviation $(\sigma), 95 \%$ of the population is kept in the chosen confidence interval.

On the globally smooth models we studied, the coefficient

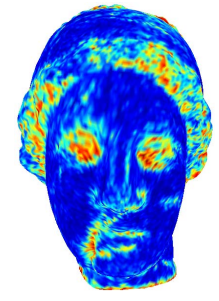

(a)

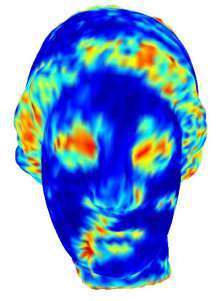

(b)

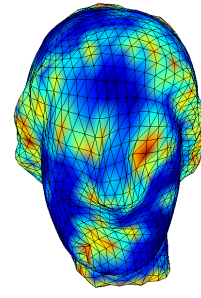

(c)

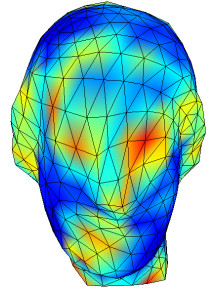

(d)
Figure 5. Gaussian normalized distributions of the wavelet coefficient norm, on the first four resolution levels of the Normal Venus model.

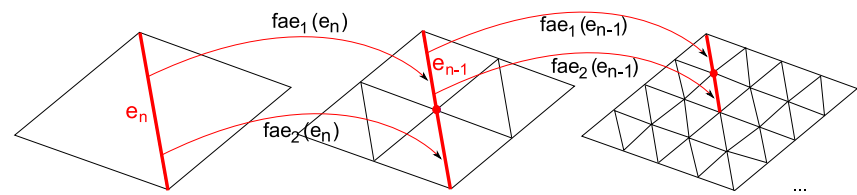

(a)

(b)

(c)

Figure 6. Wavelet "fine averaging association". For an edge $e_{n}$ in a coarse decomposition, we compute an averaging of its wavelet norm and the norms of its finer associated edges ( $f a e)$ in all the existing resolution levels.

magnitude distributions allow to identify high curvatures characterizing the eyes, nose, mouth or ears, and also the object tips (neck or feet). This spreading emphasizes also the textured parts, such as the hair of the Venus head. These experimental amplitude distributions corroborate our previous hypothesis about the faculty of the subdivision surfaces to well predict the mesh smooth parts. Moreover even in globally smooth models, there remain some areas where other considerations should be employed.

In the Horse and Venus first four resolution levels (Fig. 4 and 5), the same main features are underlined, but the fineness of these highlighted high frequencies is quite different in each level. Since we aim at producing a segmentation that identifies clearly the high frequencies lost during the coarsification process, a global consideration of all the coefficient amplitudes in the entire "hierarchy" is required. The segmentation algorithm we used in this context was conceived to apply on one mesh at a time. Consequently instead of segmenting all the approximations, we propose to compute an averaging of all the wavelet coefficient amplitudes.

We chose to segment one of the coarsest resolution levels, for each coarse triangle to be assigned a unique region number. It gives us the opportunity to independently treat each produced region, without being forced to remesh them, in the case where a subdivision connectivity is not encountered. Hence the chosen coarse level determine the number of resolution levels involved in the patch-independent MRA. Considering this setting, the averaging rule we adopted is illustrated in Fig. 6. Each edge of the coarse level is then associated to two children edges (called fae in Fig. 6) in the immediatly finer mesh. We applied a weighted averaging where each coefficient norm is multiplied by $2^{d}$, to take into account the decrease of the wavelets in the "hierarchy" (explained in Section II-A) - $d=0$ for the coarsest edges and increasing integer values for the finer decompositions.

\section{WAVELET-BASED CLASSIFICATION AND SEGMENTATION}

Given the wavelet norms, averaged on one of the coarsest decompositions, we used an extension of the algorithm developped by Lavoué et al. [20], to create a mesh segmentation. This method is based on a K-Mean classification [21]. 


\section{A. Mesh Classification and Segmentation Algorithms}

In the past twenty years, much work has been done in shape decomposition because of its utility in many computer graphic applications. These methods are usually designed to solve specific problems and are difficult to compare. Some applications use segmentation principles to simplify treatments like texture mapping, parameterization, mesh editing, deformation or compression on complex meshes with a high genus, for example. They are generally faster and more simple on surface patches homeomorphic to a disc. The partitioning criterion generally used in this setting is the discrete curvature or the planarity information. Our segmentation process use a different criterion together with an adaptation of the classification and segmentation algorithms implemented by Lavoué et al. [20].

They used a curvature tensor estimation [22] to compute the principal curvature values in each vertex. The produced segmentations delimit smooth regions surrounded by sharp edges on CAD objects. Our adaptation replaces this curvature criterion by our previously defined wavelet norm averaging.

The K-Mean classification algorithm was first used to create two groups of vertices (referred as clusters): one with the smallest amplitudes (represented in dark blue in next figures, and referred as the smooth cluster) and the other with the highest ones (in light green). After several iterations, starting with two randomly determined centroïds, each vertex is then associated to a cluster $C_{i}$ and a "roughness value" $r_{i}$. Homogeneous connex patches are then deduced with a region growing and labeling algorithm adapted from [20]. More precisely, the studied measure is transmitted from vertices to triangles, starting from seed triangles whose incident three vertices belong to the same cluster. Thanks to this framework, a mesh decomposition in a small number of regions can be created at any given resolution level.

\section{B. Segmentation results}

We illustrate in Fig. 7 to 9 the different steps involved in our segmentation process, respectively for the Horse, Venus and Skull Normal models. Colors used for the patches were randomly chosen. The arrows in the pictures serve to indicate which region will be further studied in the rest of the paper. The result of our averaging rule is depicted on meshes which have a various number of vertices (because different numbers of resolution levels were considered). It has a tendancy to stump the isolated details and sharpen the very important ones (highlighted in various resolutions). The number of created patches is fully dependent on the choice of the coarse level. We examined different choices, to study the influence of the chosen coarse level, on the further detailed experimentations.

Given the segmentations of the Normal models on a coarse level, the final stage of our pre-processing consists in projecting the coarse regions on the original SR model. This

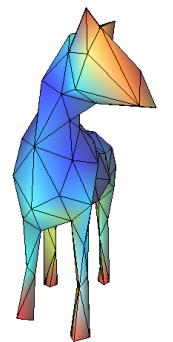

(a)

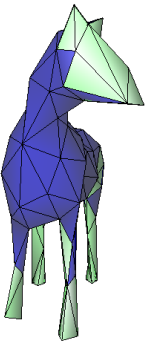

(b)

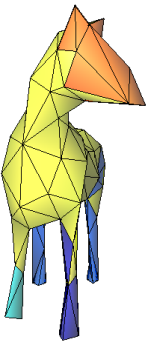

(c)

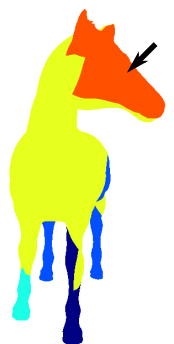

d) (e)

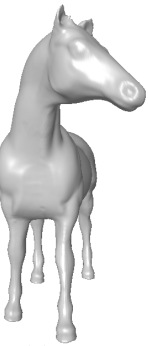

Figure 7. Illustration of the different steps involved in our classification and segmentation processes, on the $5^{t h}$ level of the Normal Horse model. (a) Averaging of the wavelet norms in the $5^{\text {th }}$ level followed by (b) the KMean classification and (c) segmentation in 5 regions; (d) Fine projection of the segmentation on the SR original model whose features can be better appreciated with a Phong shading (e).

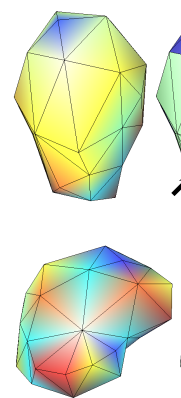

(a)
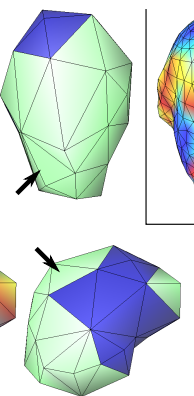

(b)

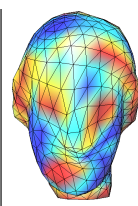

(c)

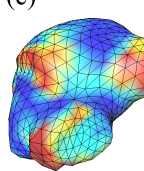

(f)

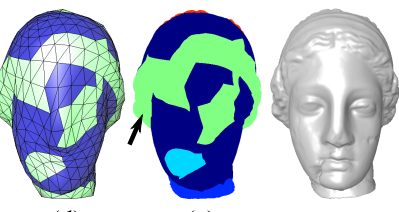

(d)

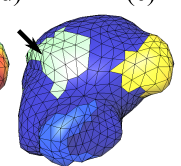

(g)
Figure 8. Illustration of the different steps involved in our classification and segmentation processes, on the $6^{t h}$ (left) and the $4^{t h}$ (right) levels of the Normal Venus model. (a, c, f) Averaging of the wavelet norms in a coarse level followed by (b, d) the K-Mean classification and (g) segmentation in 6 regions (on the $4^{t h}$ level). Three regions are obtained in the $6^{\text {th }}$ decomposition (left). (e) Fine projection of the $4^{t h}$ level segmentation on the SR original model.

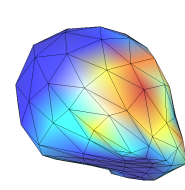

(a)

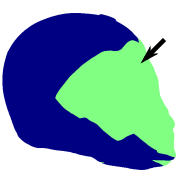

(b)

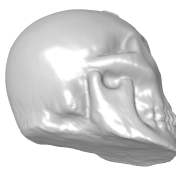

(c)

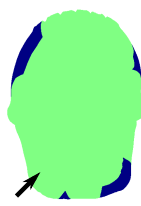

(d)

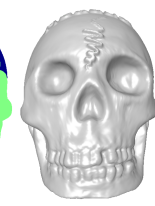

(e)
Figure 9. Illustration of the different steps involved in our classification and segmentation processes, on the $5^{t h}$ level of the Normal Skull model. (a) Averaging of the wavelet norms in the $5^{\text {th }}$ level. The segmentation in two regions is illustrated in the SR original model, whose features can be better appreciated with a Phong shading (c, e). 
stage is simply realized incrementally, resolution per resolution, where the four children facets associated to each coarse triangle are assigned the same cluster and region number as their father triangle. The resulting fine segmentations can now be the input of our patch-independent MRA, detailed in the following.

\section{REGION-BASED MRA FOR VIEW-DEPENDENT RECONSTRUCTION}

As a preliminary step of the development of a locallybased R-D optimized coding scheme, we propose to detail in this Section the three different applications of the Butterfly stencil we examined close to the patch borders, during the region-based MRA and coding.

In our first consideration (designed later by "No PB": for No Patch Borders taken into account), the unlifted Butterfly wavelet decomposition is realized as if any patch was defined. This rule is accurate if a uniform reconstruction of each patch is adopted (with approximately the same $L^{2}$ error orders, in each region). In other cases, an alteration of the quality of some regions can appear due to the influence of the "poor" reconstructions of their neighboors, for example.

For these other cases (which don't necessarily involve a uniform reconstruction of each patch, like view-dependent transmission and rendering), we propose to examine two different adaptations of the Butterfly stencil close to the patch borders. These two adaptations (designed later by "PB") will be applied when each patch is seen as an independent non-closed surface. With this latter setting, any region can influence its neighboors during the "non-uniform" region-based reconstructions.

Fig. 10 shows the amplitude and polar angle (the angle between each coefficient and its related surface normal vector) distributions for each of the three rules. We can deduce that the two predictions associated to the "PB" consideration (histograms on the middle and on the bottom) are not as good as the classical ("No PB") one (on the top), because the wavelet amplitude and polar angle distributions are generally more dispersed. The first rule we considered (close to the patch borders) uses two extensions of the classical Butterfly stencil (illustrated in Fig. 11) to predict the new vertices situated on the borders (picture $(a)$ ) or surrounded by extraordinary vertices (picture $(b)$ ), as it can appear close to the patch borders.

The goal of our new rule (designed later by "PB SYM") is to recreate the "missing coarse vertices" in the classical Butterfly stencil (illustrated in Fig. 1). These vertices are missing because we take into account the patch borders in the MRA. The position of these created "ghost vertices" is deduced from the other real coarse vertices involved in the Butterfly stencil. We illustrate in detail in Fig. 12 the concept we used for deducing them, with a tendancy to favor the diagonal symmetry. We also enumerate all the possible cases we can encounter and for the ones for which a diagonal
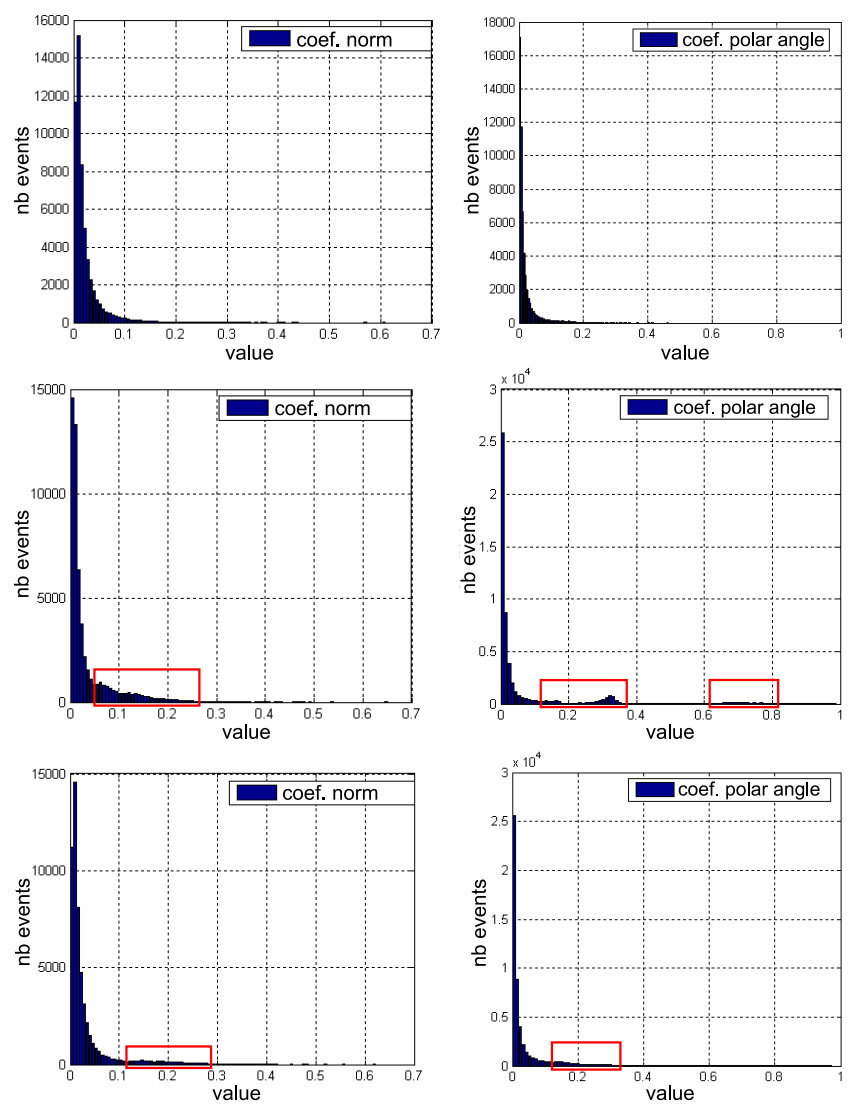

Figure 10. Amplitude (left) and polar angle (right) distributions in every resolution level of the Venus Normal segmented model (right segmentation of Fig. 8 considered). The red boxes highlight the differences noticed in the two "PB" distributions (middle: classical rule - bottom: our new rule), in comparison with the distributions for which the classical Butterfly stencil was used, without taking into account the patch borders (top).

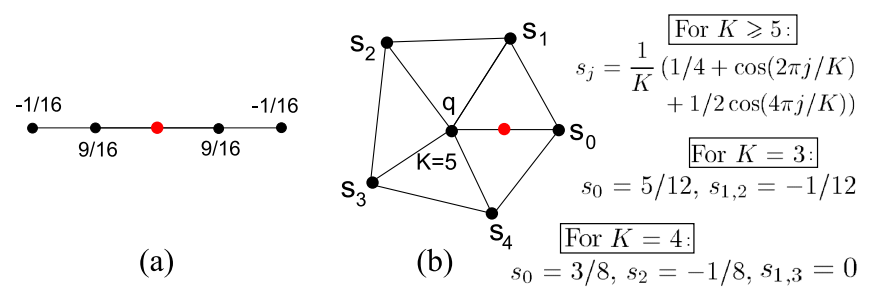

Figure 11. (a) Special Butterfly stencil used to predict the red vertex, situated on the mesh border (for non-closed 3-D meshes); (b) Extension of the classical Butterfly stencil for extraordinary vertices (scheme proposed by Zorin et al. [23]). 

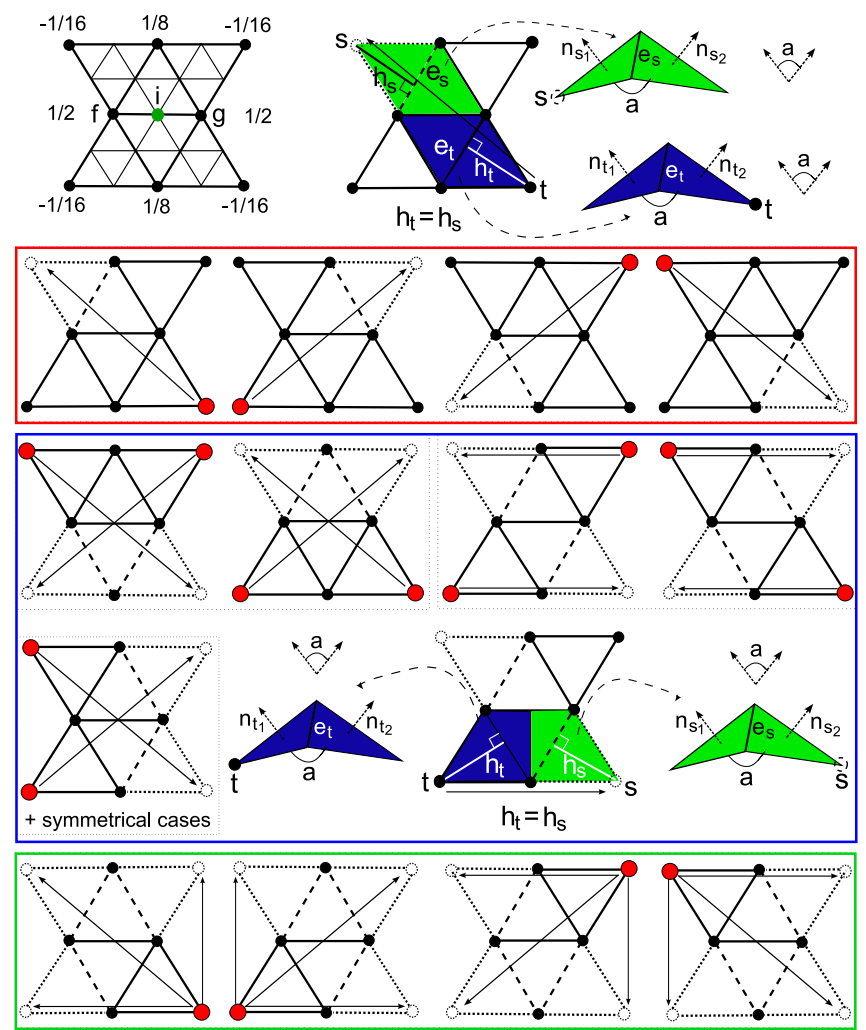

Figure 12. Definition of our new scheme, used when some of the coarse vertices are missing in the Butterfly stencil, due to patch borders (illustrated with big dashed lines). Top left: classical Butterffly stencil; Top right: method used to recreate the "missing coarse vertices" in the Butterfly stencil. We favor the diagonal symmetry (computed thanks to the red vertices in the next pictures), but for the cases where it is not possible, we define an equivalent rule (illustrated with colours in the middle of this figure). The different configurations are highlighted with big rectangles, when only a coarse vertex (top in red), two coarse vertices (middle in blue) or three coarse vertices (bottom in green) are missing.

symmetry cannot be computed, we define an equivalent rule (illustrated with colours in the middle of Fig. 12).

In Fig. 10, we can observe that our new rule distributions (on the bottom) are closer to the classical ("No PB") ones (on the top). Moreover we have the possibility to perfectly decode some regions of a mesh while other are at the same time "poorly" reconstructed.

\section{ExPERIMENTAl Results AND ApPlicAtions}

We present in this Section, various experimental results we obtained on the Normal meshes, segmented with our wavelet-based partitioning algorithm, but any other kind of SR mesh segmentation could also have been considered, like the ones implemented by Sim et al. [8] or by Payan et al. [10].

We first propose a visual comparison of the mesh distortion in Fig. 13. We compared two different "viewdependent" reconstructions of the segmented Skull model (produced by the "No PB" consideration). Hence we com-
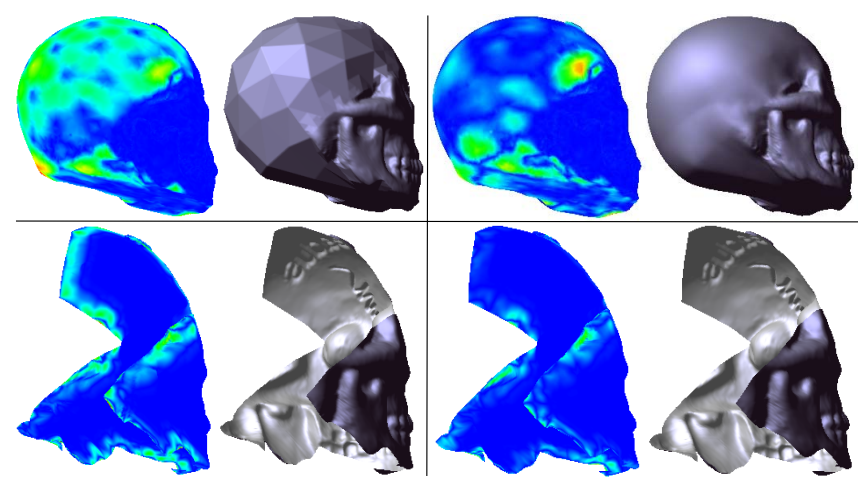

Figure 13. Mesh distortion on two different "view-dependent" reconstructions, presented on the entire mesh (top) and on the non-smooth cluster (bottom) of the Skull segmented model (illustrated in Fig. 9). Patch borders are not taken into account during the MRA (referred as the "No PB" consideration) and any wavelets are added on the smooth cluster. In the left pictures any prediction operator is considered, whereas in the right ones the Butterfly prediction is used. The colours depend on the magnitude of the local $L^{2}$ errors, from the minimal (blue) to the maximal (red) value. These pictures were realized thanks to the "Mesh" software [24].

puted the $L^{2}$ errors with the "Mesh" software [24], on the entire Skull model (in comparison with the irregular original model), and also on its non-smooth cluster. In the left part any prediction operator nor wavelets (only canonical quadrisections) were considered on the smooth cluster. Hence it can be interpreted as a non-visible part in a view-dependent consideration, whereas the other cluster is perfectly decoded. The only difference in the right part is that the Butterfly prediction was used in the smooth cluster. As expected, these pictures show that a less important distortion can be obtained with the Butterfly prediction, when patch borders are not taken into account during the MRA ("No PB").

This assumption is also confirmed by the Table I. It results from a computation of the $L^{2}$ errors for the "No PB" and "PB" considerations, with or without a Butterfly prediction on the smooth region(s). The distortion is presented for all the segmentated models we previously commented, only for one of their non-smooth regions (indicated with an arrow in Fig. 7 to 9). In each studied mesh region, the smallest $L^{2}$ errors are emphasized with bold characters. The same errors are obtained in the last two columns, because we used a "PB" consideration which doesn't depend on the prediction used in the neighbooring smooth cluster and thus gives the smallest distortions. For each model, the "No PB" consideration associated with a Butterfly prediction in the smooth cluster always gives the better results than without any prediction.

We finally present the bitrate-PSNR curves in Fig. 14, computed by only taking into account the non-smooth region of the segmented Skull model (the Butterfly prediction is used -without any wavelets- in the smooth region). The bitrate is reported with respect to the number of vertices in the semi-regular (SR) mesh. These curves were obtained 
Table I

$L^{2}$ ERRORS FOR THE "NO PB" AND "PB" CONSIDERATIONS, ON THE REGIONS INDICATED WITH AN ARROW IN FIG. 7 TO 9. ANY PREDICTION OPERATOR OR THE BUTTERFLY PREDICTION TOGETHER WITH ANY WAVELETS WERE CONSIDERED ON THE SMOOTH CLUSTER, WHEREAS THE OTHER CLUSTER WAS DECODED PERFECTLY.

'nb lev' = number of resolution levels.

'\# $V_{s r}$ ' = number of vertices in the SR mesh.

'Butt' = the Butterfly prediction was used in the smooth cluster.

\begin{tabular}{|c||c|c||c|c||c|c|}
\hline & nb lev & $\# V_{s r}$ & No PB & No PB Butt & PB & PB Butt \\
\hline Venus part. & 6 & 21,545 & $9.053 .10^{-4}$ & $4.107 .10^{-4}$ & $\mathbf{8 . 3 0 1 . 1 0 ^ { - 7 }}$ & $\mathbf{8 . 3 0 1 . 1 0 ^ { - 7 }}$ \\
\hline Venus part. & 4 & 76,193 & $1.345 .10^{-4}$ & $1.015 .10^{-4}$ & $\mathbf{1 . 4 5 6 . 1 0 ^ { - 6 }}$ & $\mathbf{1 . 4 5 6 . 1 0 ^ { - 6 }}$ \\
\hline Horse part. & 5 & 7,857 & $6.414 .10^{-5}$ & $5.616 .10^{-5}$ & $\mathbf{7 . 7 7 5 . 1 0 ^ { - 8 }}$ & $\mathbf{7 . 7 7 5 . 1 0 ^ { - 8 }}$ \\
\hline Skull part. & 5 & 56,865 & $5.260 .10^{-4}$ & $3.433 .10^{-4}$ & $\mathbf{5 . 9 9 0 . 1 0 ^ { - 7 }}$ & $\mathbf{5 . 9 9 0 . 1 0 ^ { - 7 }}$ \\
\hline
\end{tabular}

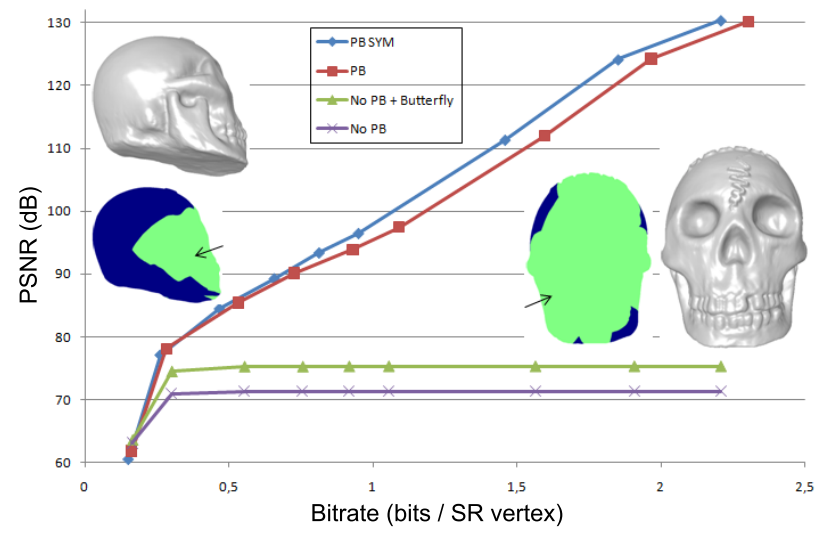

Figure 14. Bitrate-PSNR curves for the non-smooth region of the segmented Skull model, where the Butterfly prediction is used (without any wavelets) in the smooth region. The bitrate corresponds to the bit allocation after quantization in the SR non-smooth patch.

with the view-dependent geometry coding (developped by Payan et al. [10]), which we adapted to our segmentation process.

$P S N R=20 \log _{10}$ peak/d, where peak is the bounding box diagonal and $d$ the $L^{2}$ relative error, corresponding to the following $L^{2}$ distance $d(X, Y)$ between the surfaces $\mathrm{X}$ and Y:

$$
d(X, Y)=\left(\frac{1}{\operatorname{area}(X)} \int_{x \in X} d(x, Y)^{2} d x\right)^{\frac{1}{2}},
$$

where $d(x, Y)$ is the Euclidean distance from a point $\mathrm{x}$ on $\mathrm{X}$ to the closest point on $\mathrm{Y}$. The results we deduced from the Table I are confirmed by the repartition of the curves (the "PB" consideration gives the best results at any bitrate). Moreover we can notice that our new prediction scheme (associated to the "PB SYM" consideration) performs better than the other "PB" rule, for view-dependent reconstruction purposes. For the "No PB" consideration, we can see that the two curves reach quickly their asymptotic value which is linked to the $L^{2}$ errors illustrated in the bottom part of the Fig. 13.

\section{CONCLUSION AND Future WORK}

A new wavelet-based segmentation algorithm for SR meshes was introduced as a pre-processing step of a viewdependent geometry coding and transmission of 3-D scenes. Our segmentation process aims at constructing regions with different degrees of roughness, reflected by the wavelet coefficient amplitudes (associated to each resolution level). The simplification meshes were obtained with an unlifted Butterfly wavelet decomposition on Normal meshes. As a preliminary step of the development of a locally-based R$\mathrm{D}$ optimized coding scheme, we have studied the behaviour of the wavelet decomposition in the created regions, during the coding and the view-dependent reconstruction processes. We particularly investigated three different possible wavelet decompositions, close to the patch borders. One of them is a new consideration and has given the best results for viewdependent reconstruction purposes.

As an extension of the current work, some future research can be performed in the following areas. First, we may consider the patch-independent R-D optimized compression and coding. New prediction schemes and coding techniques could be proposed for the non-smooth regions (where the predictions with actual subdivision schemes can be improved), together with more subjective distortion metrics, based on human vision. Our goal will be to improve the compression bitrates on natural objects, which can be composed of non-smooth textured parts. A possible issue could consist in conceiving a statistical analysis model of the surface. The critical elements could hence serve to regenerate the same visual aspects and high frequencies of the studied surface. Another interesting consideration would be to take into account the surface anisotropy, to better predict shapes with features like sharp creases, corners or rough textures. Moreover, a fractal analysis could also be adopted in noisy regions.

We could also conceive our segmentation process differently, by better analysing the decrease of the wavelets and their relation to the surface discrete roughness or saliency. Finally other applications could benefit from our patch-based 
MRA, like watermarking. It would allow to apply different marks according to the visual aspect of the surface. Another interesting problem which can benefit from our framework are the error-resilient mesh encoding techniques.

\section{ACKNOWLEDGMENT}

I would like to address special thanks to Frédéric Payan for his R-D optimization code he gave and explained me. I would like to thank also Marc Antonini for the opportunity he offered me to work on his team in the I3S lab, in SophiaAntipolis. I finally don't forget Andrei Khodakovsky who has provided me the remeshed models used in this work.

\section{REFERENCES}

[1] H. Hoppe, "Progressive Meshes," in ACM SIGGRAPH'96, 1996, pp. 99-108.

[2] D. Cohen-Or, D. Levin, and O. Remez, "Progressive compression of arbitrary triangular meshes," in VIS'99: Proceedings of the conference on Visualization'99, 1999, pp. 67-72.

[3] R. Pajarola and J. Rossignac, "Compressed progressive meshes," IEEE Transactions on Visualization and Computer Graphics, vol. 6, no. 1, pp. 79-93, 2000.

[4] A. Khodakovsky, P. Schröder, and W. Sweldens, "Progressive geometry compression," in ACM SIGGRAPH'00, 2000, pp. 271-278.

[5] A. Khodakovsky and I. Guskov, "Compression of normal meshes," Geometric Modeling for Scientific Visualization, pp. 189-206, 2003.

[6] S. Lavu, H. Choi, and R. Baraniuk, "Geometry compression of normal meshes using rate-distortion algorithms," in SGP'03: ACM SIGGRAPH Symposium on Geometry processing, 2003, pp. 52-61.

[7] F. Payan and M. Antonini, "An efficient bit allocation for compressing normal meshes with an error-driven quantization," Computer Aided Geometric Design - Special issue on Geometry Mesh Processing, vol. 22, no. 5, pp. 466-486, 2005.

[8] J.-Y. Sim, C.-S. Kim, C. Kuo, and S. Lee, "Rate-distortion optimized compression and view-dependent transmission of 3-D normal meshes," IEEE Transactions on Circuits and Systems for Video Technology, vol. 15, no. 7, 2005.

[9] P. Gioia, O. Aubault, and C. Bouville, "Real-time reconstruction of wavelet-encoded meshes for view-dependent transmission and visualization." IEEE Transactions on Circuits and Systems for Video Technology, vol. 14, no. 7, pp. 1009-1020, 2004.

[10] F. Payan, M. Antonini, and F. Mériaux, "View-dependent geometry coding of 3D scenes," in ICIP, 2009, pp. 729-732.

[11] H. Hoppe, "View-dependent refinement of progressive meshes," in ACM SIGGRAPH'97, 1997, pp. 189-198.

[12] C. Roudet, F. Dupont, and A. Baskurt, "Multiresolution mesh segmentation based on surface roughness and wavelet analysis," in SPIE VCIP'07: Visual Communications and Image Processing, vol. 6508, no. 1, 2007, p. 65082E.
[13] N. Dyn, D. Levin, and J. A. Gregory, "A butterfly subdivision scheme for surface interpolation with tension control," $A C M$ Transactions on Graphics, vol. 9, no. 2, pp. 160-169, 1990.

[14] S. Mallat, "A theory for multiresolution signal decomposition: The wavelet representation." IEEE Trans. Pat. Anal. Mach. Intell., vol. 11, pp. 674-693, 1989.

[15] W. Sweldens, "The lifting scheme: A new philosophy in biorthogonal wavelet constructions," in Wavelet Applications in Signal and Image Processing III, 1995, pp. 68-79.

[16] M. Lounsbery, T. D. DeRose, and J. Warren, "Multiresolution analysis for surfaces of arbitrary topological type," $A C M$ Transactions on Graphics, vol. 16, no. 1, pp. 34-73, 1997.

[17] A. W. F. Lee, W. Sweldens, P. Schröder, L. Cowsar, and D. Dobkin, "MAPS: multiresolution adaptive parameterization of surfaces," in ACM SIGGRAPH'98, vol. 32, 1998, pp. 95-104.

[18] I. Guskov, K. Vidimce, W. Sweldens, and P. Schröder, "Normal meshes," in ACM SIGGRAPH'O0, 2000, pp. 95-102.

[19] I. Guskov, "Manifold-based approach to semi-regular remeshing," Graph. Models, vol. 69, no. 1, pp. 1-18, 2007.

[20] G. Lavoué, F. Dupont, and A. Baskurt, "A new CAD mesh segmentation method, based on curvature tensor analysis," Computer-Aided Design, vol. 37, no. 10, pp. 975-987, 2005.

[21] A. Gersho and R. M. Gray, Vector quantization and signal compression. Kluwer Academic Publishers, 1991.

[22] D. Cohen-Steiner and J.-M. Morvan, "Restricted Delaunay triangulations and normal cycle," in SCG'03: Symposium on Computational Geometry, 2003, pp. 312-321.

[23] D. Zorin, P. Schröder, and W. Sweldens, "Interpolating subdivision for meshes with arbitrary topology," in ACM SIGGRAPH'96, 1996, pp. 189-192.

[24] N. Aspert, D. Santa-Cruz, and T. Ebrahimi, "MESH: Measuring errors between surfaces using the hausdorff distance," in ICME'02: IEEE International Conference on Multimedia and Expo, vol. I, 2002, pp. 705-708. 